\title{
Analysis on influencing factors and simulation of sediment release by density current: the case of Xiaolangdi Reservoir
}

\author{
Wang Ting ${ }^{1,2}$, Wang Yuanjian ${ }^{1,2, *}$, Ma Huaibao ${ }^{1,2}$, Qu Shaojun ${ }^{1,2}$ \\ ${ }^{1}$ Yellow River Institute of Hydraulic Research, Zhengzhou 450003, China; \\ ${ }^{2}$ Key Laboratory of Yellow River Sediment Research, MWR, Zhengzhou 450003, China) \\ Author introduction: Wang Ting (1980-), born in Xixian, Henan, Master's degree, senior engineer, mainly engaged in the research and \\ application pertaining to reservoir sediment and river dynamics.
}

\begin{abstract}
Density current venting by bottom outlets is the main form of sediment release from large reservoirs during sediment retention periods. Taking Xiaolangdi Reservoir as a case study, this study analyzed influencing factors of sediment release by density currents and proposed a semi-empirical formula to simulate the density current release process. The results show that the amount of incoming water and sediment, length of backwater, and sedimentation volume upstream of backwater zones are the main factors influencing sediment discharge. However, the importance of influencing factors varies slightly for different sediment supply areas. The semi-empirical formula provides a good simulation of the actual sediment release process under a relatively stable water level, and thus, it can extend scientific and technical support necessary for reservoir operation.
\end{abstract}

\section{Introduction}

The sediment release methods for reservoirs include damming release and emptying release [1-3]. Damming release is typically categorized as open-flow damming release and density current damming release. Open-flow damming release includes sediment release under high and low damming conditions. The former refers to sediment release during the reservoir water storage period, while the latter refers to that under significant water level reduction in the reservoir accompanied by a high flow rate. When the water level in front of the reservoir dam is not reduced, density current performs better than open flow in transporting sediment to the area in front of the dam [4]. The sediment release effect of the reservoir is related to the length and shape of the reservoir, gradient of the reservoir bottom, amount of incoming water and sediment, and elevation and scheduling of the releasing facilities in front of the dam [3, 5-13]. Thus far, many studies have focused on calculations regarding sediment release in reservoirs. The calculation methods differ depending on the type of sediment release. Specifically, Tsinghua University, Shaanxi Institute of Water Sciences, Yellow River Institute of Hydraulic Research, Zhang Qishun, Cao Ruxuan, and Zhang Cuiping [14-19] have conducted indepth research on the calculation of on-way scouring and headcut scouring based on their respective analyses of measured data. Fan Jiahua [20] has conducted in-depth research on the calculation and application of density currents. Zhang Junhua et al. [21-26] regulated the water and sediments of Yellow River several times based on the sediment transport characteristics of density currents, achieved satisfying results, and expanded the scope of research regarding density currents. In this study, Xiaolangdi Reservoir is considered as the research subject, and the factors influencing sediment release during the sediment retention period are analyzed. Then, based on the reservoir boundary conditions, the sediment release process is simulated using the currently available empirical formula.

\section{Region and data}

\subsection{Overview of Xiaolangdi Reservoir}

Xiaolangdi Water Control Complex is a pivotal project that focuses on flood control (ice flood control) and siltation reduction, and provides several benefits such as water supply, irrigation, power generation, decontamination, and integrated utilization. It occupies an important strategic position in the overall management and development of Yellow River. Xiaolangdi Reservoir is located in a canyon zone that is long, curved, and narrow, and contains numerous tributaries, as shown in

\footnotetext{
*Corresponding author: wangyuanjian@hky.yrcc.gov.cn
} 
Figure 1. Moreover, Xiaolangdi Reservoir dam is located $130 \mathrm{~km}$ upstream of Sanmenxia Dam. Its controlled drainage area covers $694,000 \mathrm{~km}^{2}$, accounting for $92.3 \%$ of the Yellow River Basin, and the 275-m-high reservoir has a storage capacity of 12.75 billion $\mathrm{m}^{3}$. It intercepted the Yellow River in October 1997, stored water in October 1999, and was officially put into use in May 2000.

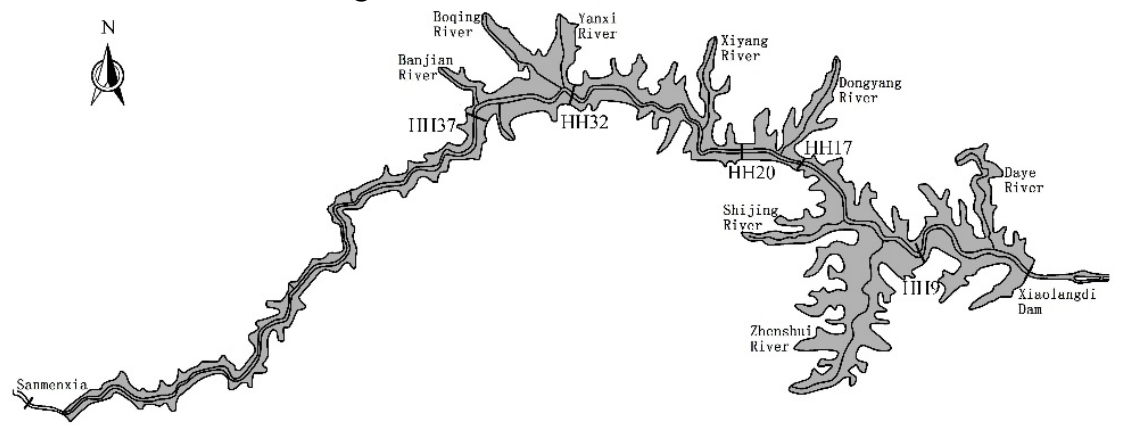

Fig. 1. Area served by Xiaolangdi Reservoir.

\subsection{Deposition patterns of the mainstream reservoir}

At the beginning of the operation of Xiaolangdi Reservoir, it mainly retained sediment and discharged clear water. By October 2000, the longitudinal section of the main stream had formed a delta deposition pattern [27] (Figure 2). Thereafter, the delta deposition pattern has been constantly adjusted, given the effects of incoming water and sediment, reservoir operation and scheduling, and reservoir topography. Typically, the delta surface has continued to rise, and the delta apex has continued to advance toward the area in front of the dam. By October 2016 , the delta apex had moved from the HH40 section located $69.39 \mathrm{~km}$ away from the dam (in October 2000) to the HH11 section located $16.39 \mathrm{~km}$ away from the dam, moving $53 \mathrm{~km}$ downstream, and the elevation of the delta apex was $222.59 \mathrm{~m}$.

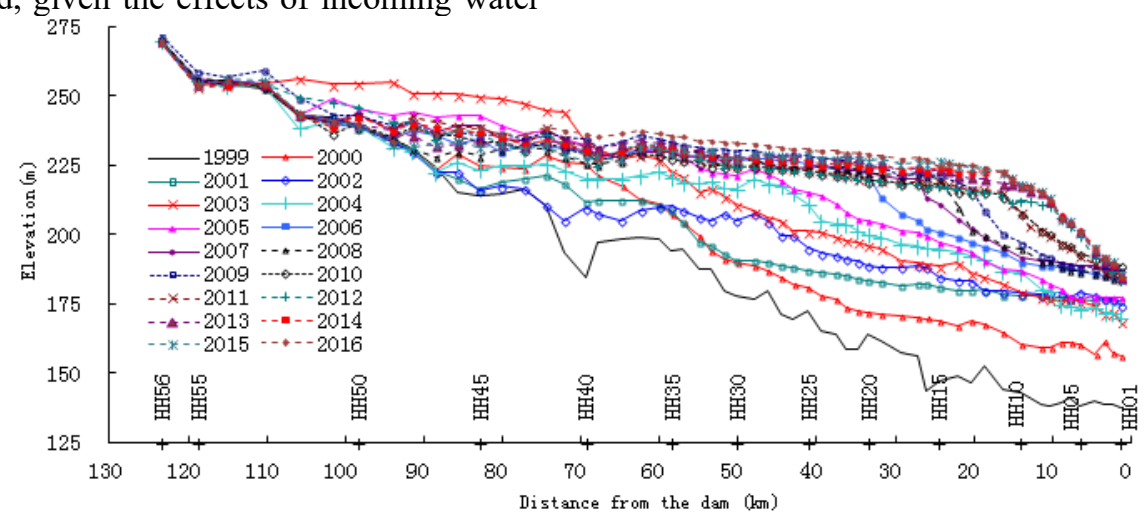

Fig. 2. Longitudinal section of the mainstream in Xiaolangdi Reservoir from 1999 to 2016 (Thalweg point after the flood season).

\subsection{Incoming/released water and sediment, and sediment deposition in the reservoir}

The Yellow River has been dry and less sediment has been recorded therein since the start of the operation of Xiaolangdi Reservoir, as shown in Figure 3. Xiaolangdi Reservoir has stored water since September 1999. Up to October 2016, the annual average incoming water flow

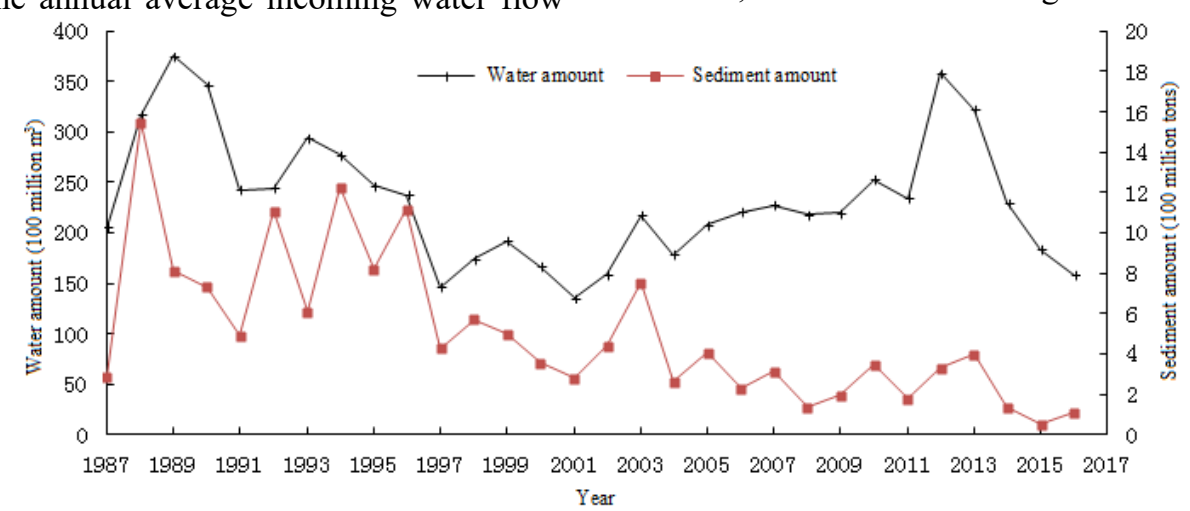

Fig. 3. Change in incoming water and sediment from 1987 to 2016 at Sanmenxia Hydrometric Station. and sediment were 21.721 billion $\mathrm{m}^{3}$ and 290.4 million $\mathrm{t}$, respectively. The amount of water was $14 \%$ lower than the 1987-1999 series since the start of the operation at Longyangxia. Further, the amount of sediment decreased by as much as $63 \%$; notably, at stage one of the late sediment retention period in 2007, the amount of incoming sediment in Xiaolangdi Reservoir decreased further, and the annual average was 219.9 million $\mathrm{t}$. 
Xiaolangdi Reservoir has stored water since September 1999. Up to October 2016, the amounts of incoming sediment, released sediment, sediment deposited in the reservoir, and annual average deposited sediment were 4.9371 billion t, 1.0381 billion t, 3.899 billion $\mathrm{t}$, and 229.4 million $\mathrm{t}$, respectively. Given the effects of the incoming water and sediment, and the operation mode of the reservoir, the difference in the annual deposited sediment is considerable, the maximum and minimum values being 635.8 million $t$ (in 2003) and 50.1 million $\mathrm{t}$ (in 2015), respectively. However, in general, sediment deposition was significant in the years that recorded high amounts of incoming sediment (Figure 4).

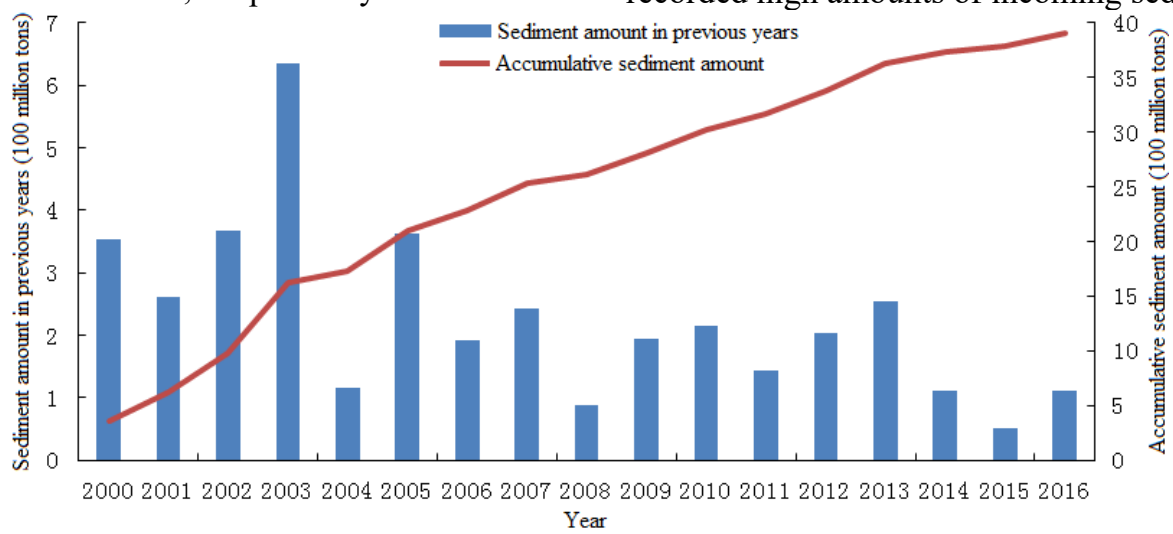

Fig. 4. Sediment amounts in Xiaolangdi Reservoir since the start of operation.

By October 2016, the total amount of released sediment from Xiaolangdi Reservoir had reached 1.0381 billion $t$ (Figure 5). Depending on the incoming water and

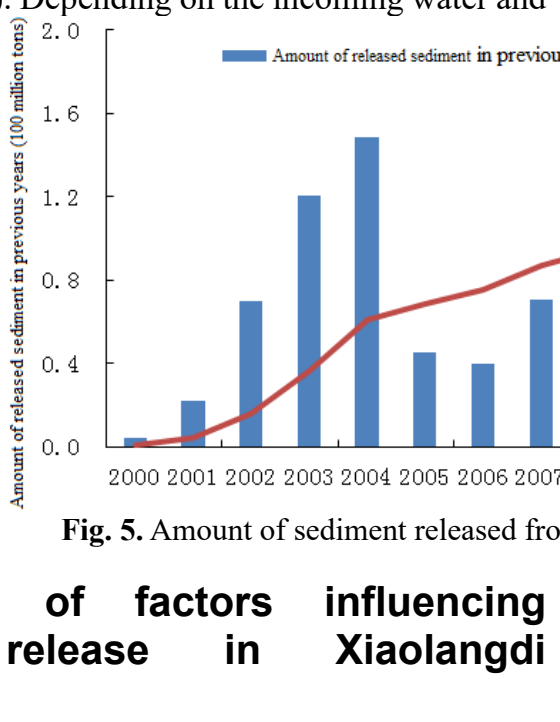

\section{Analysis of factors influencing sediment release in Xiaolangdi Reservoir}

The main form of sediment release from Xiaolangdi Reservoir is density current release during the flood season or muddy water reservoir release formed by density currents. Since the start of its application, 19 experiments on water and sediment regulation and production practices have been carried out through the scientific and rational regulation of the reservoir. Moreover, density currents have been successfully formed in Xiaolangdi Reservoir and 619.7 million $\mathrm{t}$ of sediment have been released from it, accounting for $60 \%$ of the total amount of released sediment from the reservoir. Specifically, 322.3 million $\mathrm{t}$ of sediment have been released from the reservoir in response to the 12 water and sediment regulations passed before the flood season, accounting for $52.0 \%$ of the water and sediment sediment, and the operation mode of the reservoir, the annual sediment release has differed considerably, with a maximum of 148.7 million $t$ (in 2004) to zero sediment release from the reservoir in 2015 and 2016.

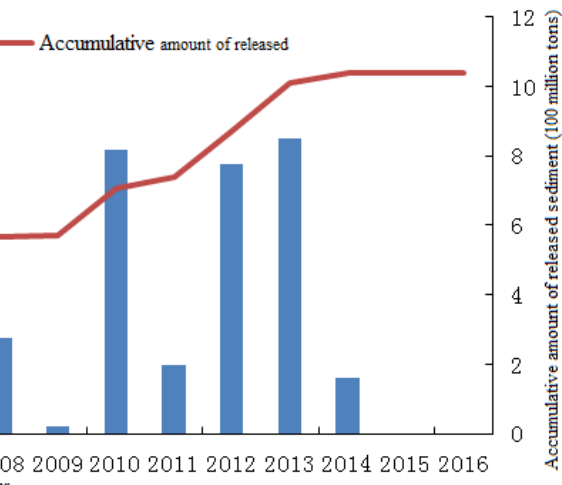

regulation amount and translating to a sediment release ratio of $60.9 \%$. Additionally, 297.4 million $t$ of sediment has been released from the reservoir in response to the seven water and sediment regulations before the flood season, accounting for $48.0 \%$ of the water and sediment regulation amount and resulting in a sediment discharge ratio of $45.6 \%$. During the water and sediment regulation period, the sediment released from Xiaolangdi Reservoir by density currents consists of two parts. The first part relates to the sediment from Xiaolangdi Reservoir itself, namely the sediment above the backwater end of Xiaolangdi Reservoir, scoured by the clear water discharge process in Sanmenxia Reservoir. The second part relates to the sediment outside Xiaolangdi Reservoir, namely that in Sanmenxia Reservoir, scoured by the midstream natural flood or the discharge flow process of Wanjiazhai Reservoir [28]. Hereinafter, the relationship between the sediment releasing effect of Xiaolangdi Reservoir and various influencing factors is analyzed based on the aforementioned sources of sediment. 


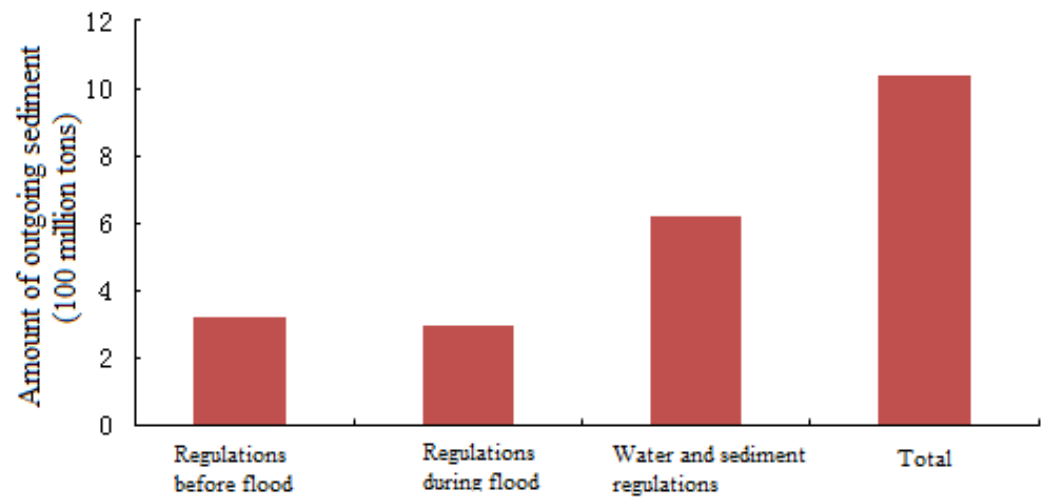

Fig. 6. Comparison of sediment releasing in different periods in Xiaolangdi Reservoir from 2000 to 2016.

open-flow uniform sediment transport, damming openflow sediment transport, and density current sediment

\subsection{Sediment from Xiaolangdi Reservoir}

The sediment above the backwater end of Xiaolangdi Reservoir, scoured by the clear water discharge process of Sanmenxia Reservoir, forms the density current in Xiaolangdi Reservoir. In this case, the density current and sediment release mainly depend on the water storage in the previous period and drainage process of Sanmenxia Reservoir, incoming water from the Tongguan section, length of backwater of Xiaolangdi Reservoir, topographic conditions in the previous period, etc.

During the shaping of the density current, the sediment transport states in Xiaolangdi Reservoir typically include

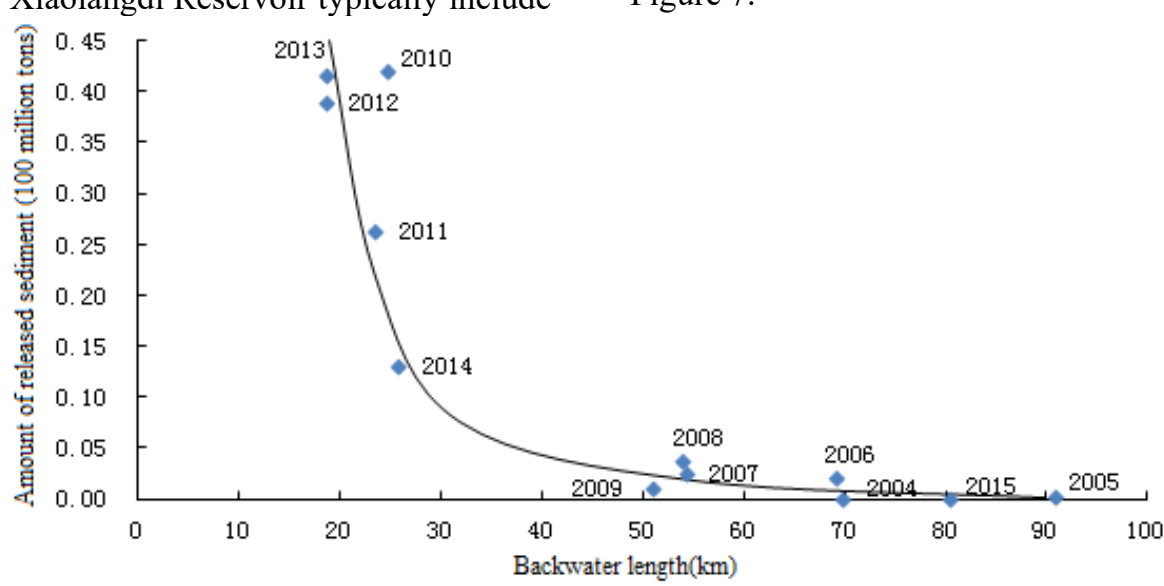

Fig. 7. Correlation between the amount of released sediment and backwater length.

When the sediment released from Xiaolangdi Reservoir is the only sediment deposited in the previous period, the amount of released sediment is correlated not only with the length of backwater but also with the topographic conditions in the previous period. The distribution of the sediment in the reservoir can clearly exhibit the topography, whereas the amount of sediment transport. When the backwater of the reservoir is longer, the scouring along the open-flow section will reduce, the damming open-flow sediment transport distance will increase, the density current submergence conditions will weaken, and the density current sediment transport distance will increase, thus reducing the sediment discharge ratio of the reservoir, and even preventing the release of some sediment from it. For the sediment from Xiaolangdi Reservoir alone, the negative correlation between the amount of sediment released from the reservoir by the density current and the length of the reservoir backwater is particularly obvious, as shown in Figure 7.

above the backwater can well reflect the sediment that can be eroded in connection to Xiaolangdi Reservoir. The data show that the amount of sediment released from Xiaolangdi Reservoir is positively correlated with the amount of sediment above the backwater, which means that as the amount of released sediment is rises with the increase in the amount of sediment, as shown in Figure 8. 


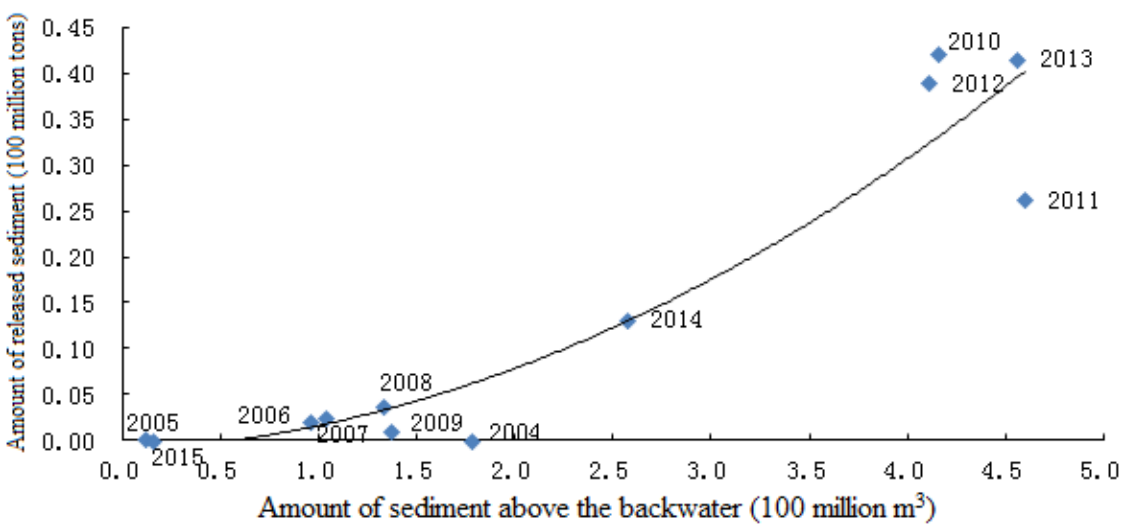

Fig. 8. Correlation between the amount of released sediment and the amount of sediment above the backwater.

in 2007, 2008, and 2009, and 2010-2013, as shown in

Density current sediment release is also closely correlated with the amount of incoming water. When the length of the backwater is equal to the amount of sediment above the backwater, the amount of released sediment increases as the amount of incoming water rises, such as
Figure 9. From 2004 to 2006 and in 2015, the length of the backwater exceeded $70 \mathrm{~km}$, and thus, more sediment above the backwater could be scoured and less sediment was released.

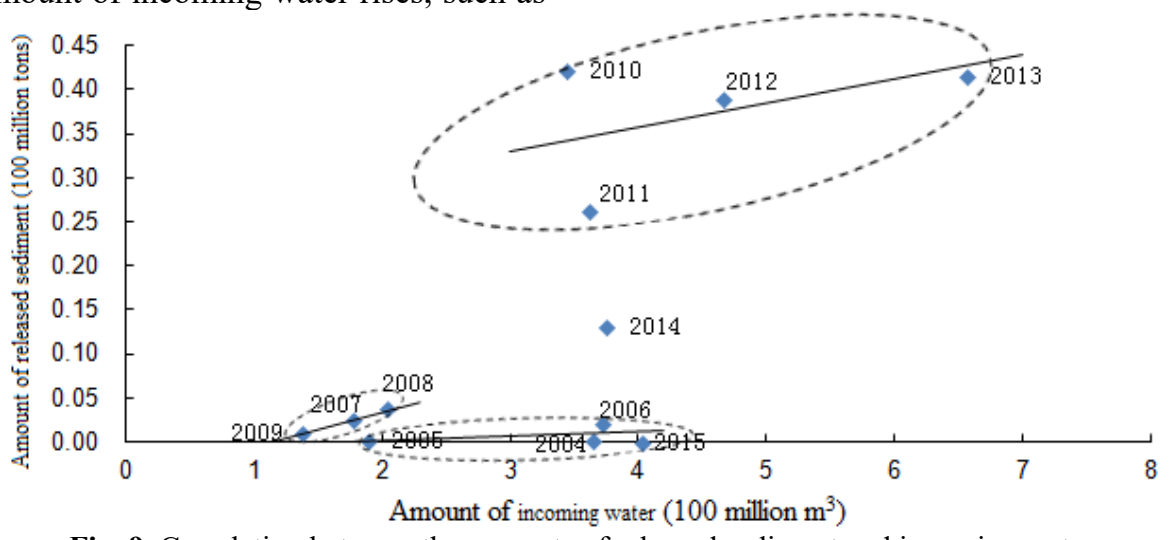

Fig. 9. Correlation between the amounts of released sediment and incoming water.

In summary, in order to facilitate better release sediment under the density current generated by the incoming sediment from Xiaolangdi Reservoir, the sediment transport distance by damming should be reduced and the amount of sediment above the backwater should be increased. In addition, it is necessary to increase the amount of incoming water.

\subsection{Sediment from outside Xiaolangdi Reservoir}

The sediment in Sanmenxia Reservoir scoured by the midstream natural flood or the discharge flow process of
Wanjiazhai Reservoir, in response to the water and sediment regulations before the flood season, forms the density current in Xiaolangdi Reservoir. In this case, the density current and sediment release mainly depend on the incoming water from Tongguan, water level control of Sanmenxia Reservoir, and operational water level of Xiaolangdi Reservoir.

During the sediment release stage of Sanmenxia Reservoir, the relatively longer backwater distance of Xiaolangdi Reservoir will increase the damming openflow sediment transport distance, extend the density current migration distance, and finally reduce the sediment releasing ratio of the reservoir or stop the density current halfway, as shown in Figure 10.

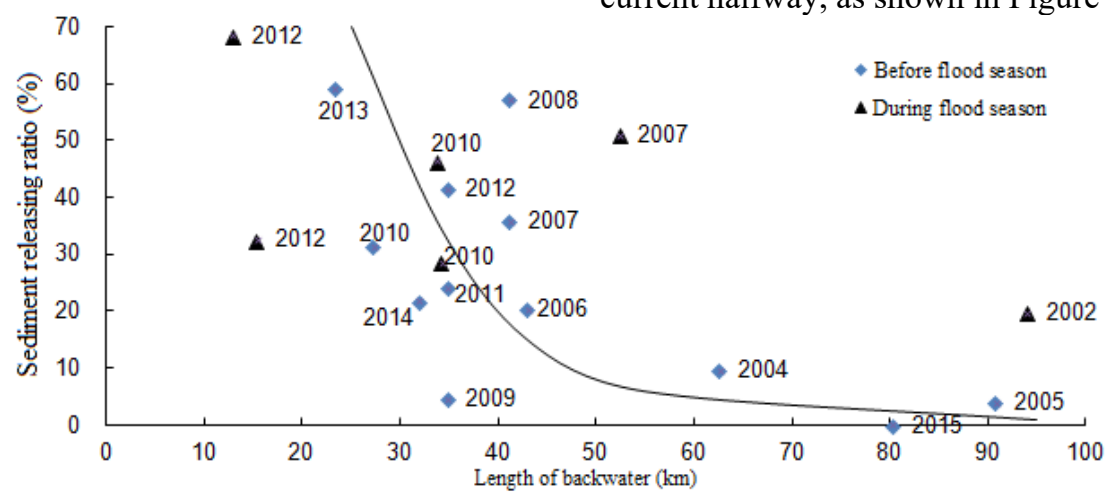

Fig. 10. Correlation between the sediment release ratio and length of backwater. 
As the amount of incoming water increases, the sediment release ratio of Xiaolangdi Reservoir increases.

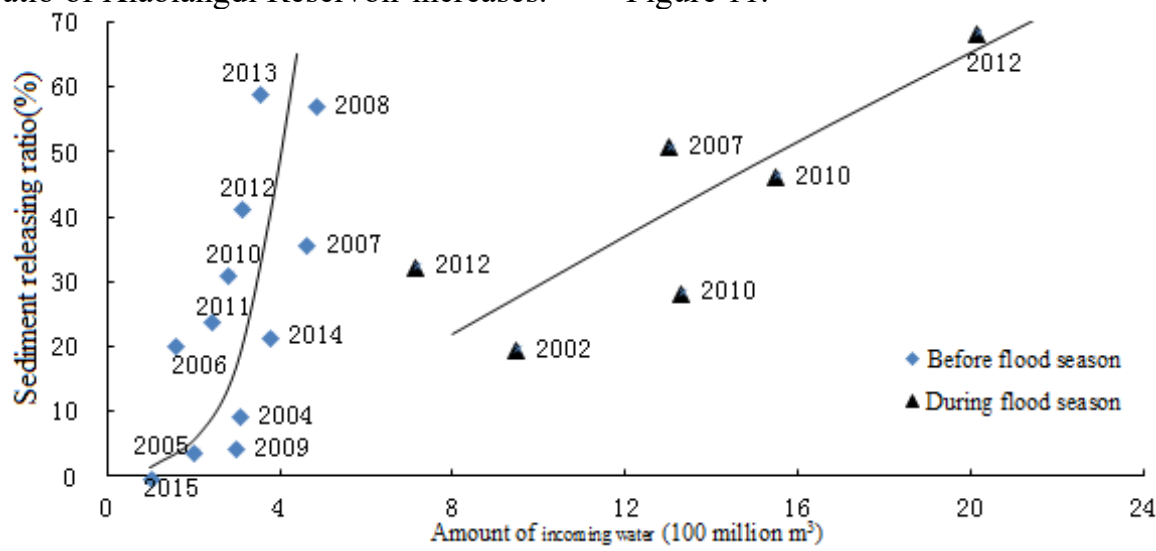

Fig. 11. Correlation between the sediment release ratio and amount of incoming water.

The sediment release ratio is positively correlated with the amount of incoming sediment (Figure 11). In 2002, 2004, 2005, and 2015, the impact of backwater on sediment release far exceeded that of other factors due to the long backwater distance. Therefore, the sediment release ratio was relatively lower. In contrast, in 2013, the

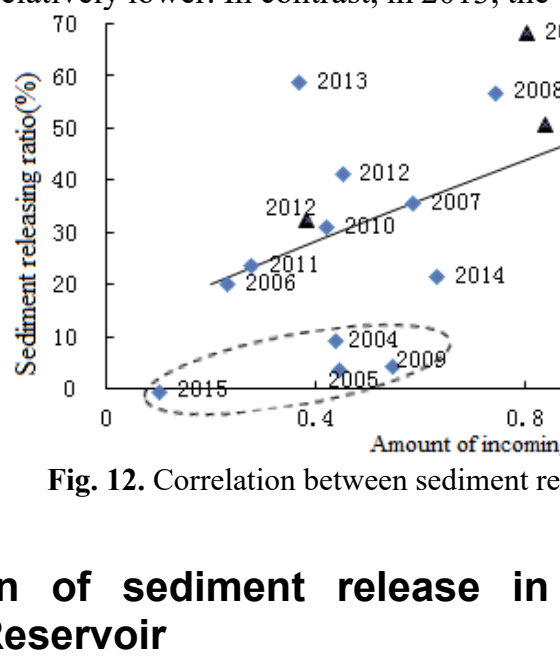

The measured water and sediment process of Sanmenxia Reservoir and existing calculation methods can be used to simulate the sediment release process of Xiaolangdi Reservoir in order to predict its sediment release effect during the flood period.

\subsection{Calculation formula}

According to the movement of water and sediment in Xiaolangdi Reservoir, the open-flow uniform sediment release (headcut scouring) and the damming open-flow sediment release of the delta are considered separately, and the density current release is calculated using the density current release formula proposed by Han Qiwei and the measured data in Xiaolangdi Reservoir [29].

(1) Open-flow scouring calculation [14]

$$
G=\psi \frac{Q^{1.6} J^{1.2}}{B^{0.6}} \times 10^{3}
$$

\section{Xiaolangdi Reservoir}

However, more water is required in the flood season to achieve the same sediment release ratio, as shown in Figure 11 .

backwater was relatively shorter, and the sediment release ratio was relatively higher; in 2009 and 2014, the larger flow led to a relatively shorter duration, resulting in a relatively lower amount of released sediment and sediment release ratio. 
$0.05 \mathrm{~mm})$, and fine sediment $(\mathrm{d}<0.025 \mathrm{~mm})$ are established using the measured data of Sanmenxia Reservoir from 1963 to 1981 and Yanguoxia from 1964 to 1969 . $\mathrm{P}_{\text {Incoming coarse sediment }}$ and $\mathrm{P}_{\text {Incoming medium sediment }}$ are the amounts of incoming coarse sediment and medium sediment, respectively.

1) Released transport rate of coarse sediment

$$
\begin{aligned}
& Q_{\text {Released coarse sediment }} \\
& =Q_{\text {Incoming coarse sediment }}\left(\frac{Q_{\text {Re seasedse dim ent }}}{Q_{\text {Inco min gse dim ent }}}\right)^{\frac{0.399}{\text { P.ncoming coarse sediment }^{1.78}}}
\end{aligned}
$$

2) Released transport rate of medium sediment

$$
\begin{aligned}
& Q_{\text {Released medium sediment }} \\
& =Q_{\text {Incoming medium sediment }}\left(\frac{Q_{\text {Released sediment }}}{Q_{\text {Incoming sediment }}}\right)^{\frac{0.0145}{\mathrm{P}_{\text {Incoming mediun sediment }}^{3.435}}}
\end{aligned}
$$

3) Released transport rate of fine sediment

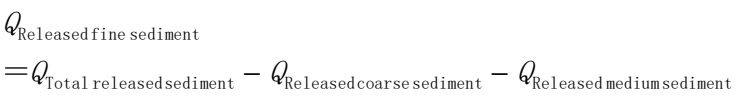

4) Density current release calculation [4]

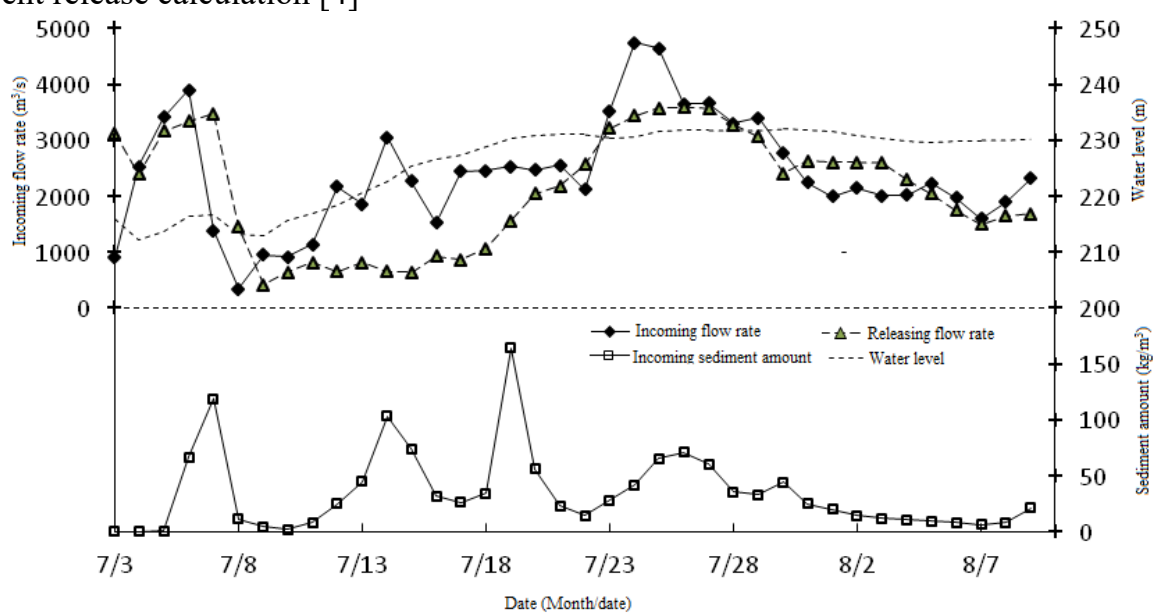

Fig. 13. Amounts of incoming and released water and sediment, and water level of Xiaolangdi Reservoir from July 3 to August 9 ,

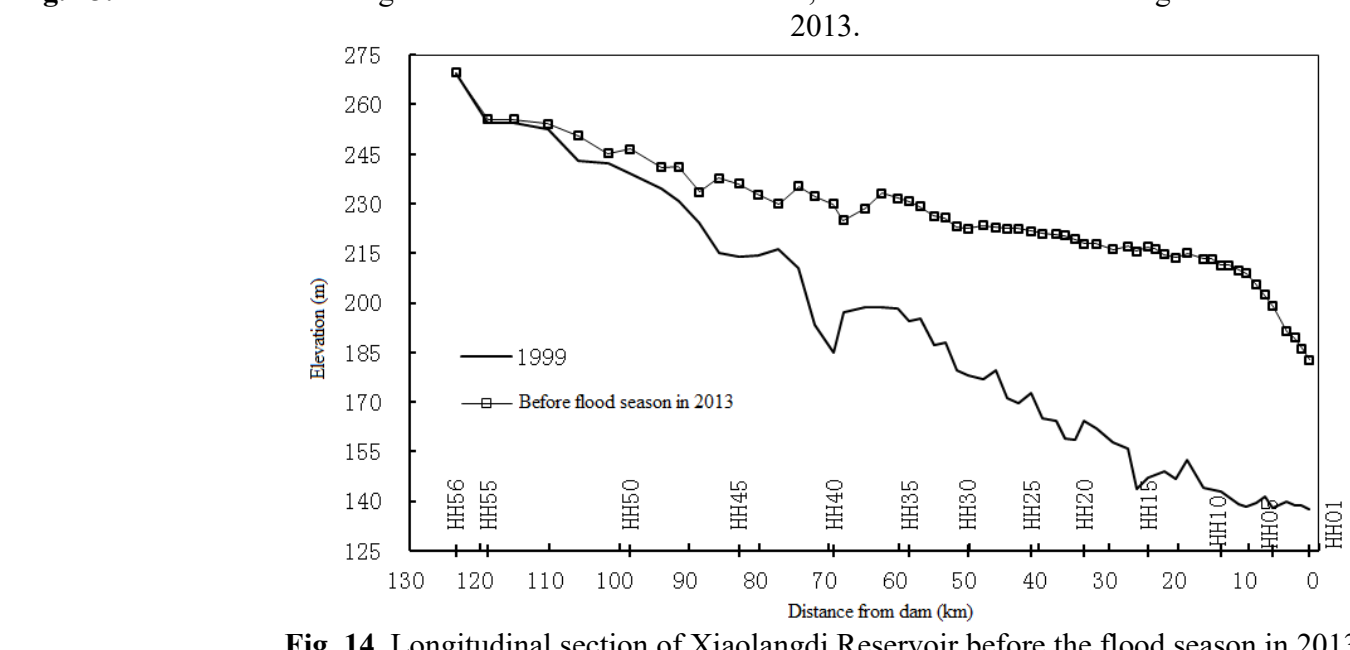

Fig. 14. Longitudinal section of Xiaolangdi Reservoir before the flood season in 2013. where $P_{4,1, i}$ is the gradation percentage of the submerged section, $\alpha$ is the saturation coefficient, $l$ is the number of particle size group, and $\omega_{l}$ is the settling velocity of $l^{l}$-th particle size group.

\subsection{Process simulation}

Water and sediment conditions and water level control: (1) The water and sediment process and operational water level from July 3 to 5, 2013 during the water and sediment regulation period before the flood season are used to simulate the open-flow and density current release process in Xiaolangdi Reservoir. (2) The water and sediment process and operational water level from July 19 to August 9, 2013 during the water and sediment regulation period are used to simulate the damming openflow sediment transport and density current sediment release process in Xiaolangdi Reservoir. The relevant amounts of water and sediment are shown in Figure 13.

Topographic conditions: The topography before the flood season in 2013 is selected as the calculation condition, as shown in Figure 14. 
Formula (1) is used to calculate the scouring of the surface and the front slope of the delta from July 3 to 5 , the result being 52.9 million t. Formula (3) is used to calculate the density current release of Xiaolangdi Reservoir, returning a result of 27 million $t$. The amount of released sediment from the reservoir is shown in Figure 15 (the time of transport from Sanmenxia to Xiaolangdi is assumed to be $24 \mathrm{~h}$ ).
Formula (2) is used to calculate the damming openflow sediment transport on the delta surface from July 20 to August 9, and formula (3) is used to calculate the density current release associated with the surface and front slope of the delta, providing a result of 66.1 million $t$. The amount of sediment released from the reservoir is shown in Figure 16.

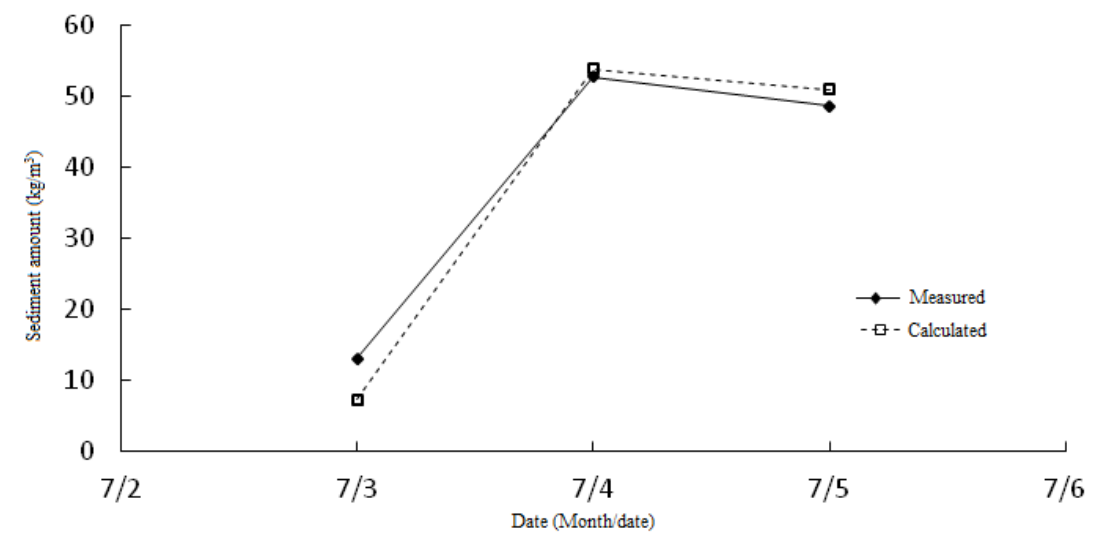

Fig. 15. Comparison of sediment amounts (using formula 2).

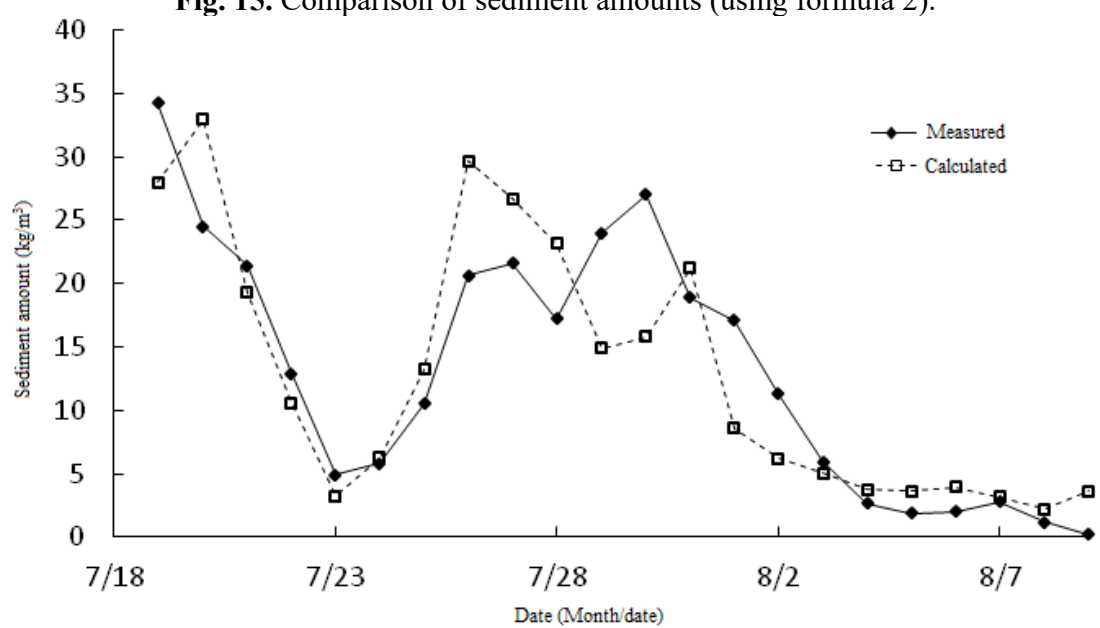

Fig. 16. Comparison of sediment amounts (using formula 2).

A comparison between the calculation results and the actual measurements from July 3 to 5 shows that the measured amount of released sediment is 27.7 million $\mathrm{t}$ and the calculated amount of released sediment is 27 million t. From July 19 to August 9, the measured and calculated amounts of released sediment are 65.8 million $\mathrm{t}$ and 66.1 million $\mathrm{t}$, respectively. The calculation results show that the simulation results of the sediment release process in the reservoir is relatively close to the measured values when the reservoir water level is relatively stable.

\section{Conclusions}

Density current release and muddy water reservoir release due to density are the main forms of sediment release for large-scale reservoirs during sediment retention periods. In the case of Xiaolangdi Reservoir, this study analyzes the factors influencing density current release for Xiaolangdi Reservoir for different sediment sources during the sediment retention period. The conclusions are as follows.

(1) The amount of incoming sediment from Xiaolangdi Reservoir, amount of incoming water, length of backwater, and sediment above the backwater are key factors affecting the sediment release of the reservoir; the sediment releasing effect of the reservoir is positively correlated with the amount of incoming water in the reservoir and the sediment above the backwater, and negatively correlated with the length of the backwater.

(2) The amount of incoming sediment from outside Xiaolangdi Reservoir, amount of incoming water, amount of incoming sediment, and length of backwater are key factors affecting the sediment release of the reservoir. The sediment releasing effect of the reservoir is positively correlated with the amounts of incoming water and incoming sediment, and negatively correlated with the length of the backwater.

(3) Depending on the boundary conditions of Xiaolangdi Reservoir and the sediment regulation period before the flood season in 2013, empirical formulae are used to calculate the sediment releasing process of 
Xiaolangdi Reservoir. It is considered that the empirical formulae can simulate the actual process during relatively stable stages of the reservoir water level, and thus, they can be used to predict the sediment releasing effect of the reservoir to a certain extent. These findings provide scientific and technical support for reservoir regulation.

\section{Acknowledgment}

Project Funding: Funded by the National Key Research and Development Program (2018YFC0407401-01), National Natural Science Foundation (51509100, 51509102, 51539004, and 51509103), and the Basic Scientific Research Funding of Yellow River Institute of Hydraulic Research (HKY-JBYW-2016-10 and HKYJBYW-2018-18).

\section{References}

1. X.Y. Guo, H.X. Song, S.Y. Liu, et al., Research on key technologies of sediment control in Bajiazui Reservoir and sediment-laden reservoirs (Publisher name, Publisher location, 2007)

2. Q.H. Tu, L.F. Yang, Sediment design manual (Publisher name, Publisher location, 2006)

3. Y. Li, Research on Key Technologies of water and sediment risk regulation in flood-frequent regions in mid-downstream of Yellow River (Publisher name, Publisher location, 2013)

4. Q.W. Han, Reservoir deposition (Science Press, Location of the publisher, 2003)

5. X.Y. Wan, Research on similarity based water and sediment scheduling of sediment-laden reservoirs (Publisher name, Publisher location, 2013)

6. T. Wang, Y.P. Ma, J.H. Zhang, et al., Yellow River 36, 4-6 (2014)

7. T. Wang, J.H. Zhang, G.M. Gao, et al., International Forum on Energy, Environment Science and Materials 1538-1543 (2015)

8. S.K. Chen, H.B. Ma, J.H. Zhang, et al., Yellow River 33, 18-20 (2011)

9. C.P. Zhang, C. Zhang, X.Y. Yi., Research on key technologies of open-flow releasing and precipitation scouring of Xiaolangdi Reservoir (Yellow River Institute of Hydraulic Research, location of the publisher, 2009)

10. T. Sumi, 4th International Conference on Scour and Erosion 608-613 (2008)

11. N. Bi, Z. Yang, H. Wang, C. Xu, Z. Guo, Catena 121, 232-240 (2014)

12. T.L. Last name, J.H. Zhang, J.Q. Xia, Abbreviated journal title 27, 716-725 (2016)

13. T. Li, J.H. Zhang, G.M. Gao, et al., Appl. Mech. Mater. 641/642, 175-178 (2014)

14. River Channel Research Office of Shaanxi Institute of Water Conservancy, Sediment Research Office of Department of Hydraulic Engineering of Tsinghua
University. Reservoir sediment (Water Resources and Electric Power Press, location, 1979)

15. Q.H. Tu, S.Y. Li, B.L. Meng, et al., Calculation method of sediment deposition in large reservoirs. Volume 4: Compilation of Yellow River sediment research reports (Publisher name, Publisher location, 1980)

16. Q.S. Zhang, Z.Q. Zhang, J. Sediment Res. 1, 1-13 (1982)

17. R.Z. Peng, J. Sediment Res. 1, 14-29 (1981)

18. J. Ju, J. Sediment Res. 1, 30-39 (1990)

19. Y.P. Wang, J.H. Zhang, P.Q. Liu, 4th national hydraulic and water conservancy information conference (Xi'an Jiaotong University Press, Xi'an, 2009)

20. J.H. Fan, Experiment and design of density current and sediment engineering (Publisher name, Publisher location, 2012)

21. J.H. Zhang, H.B. Ma, J.Q. Xia, et al., J. Hydraul. Eng. 49, 62-71 (2018)

22. J.H. Zhang, T. Wang, S.Q. Jiang, et al., Proceedings of ISRS 602-608 (2013)

23. J.H. Zhang, H.B. Ma, S.T. Dou, et al., Proceedings of the 5th international Yellow River forum on ensuring water right of the river's demand and healthy river basin maintenance (Yellow River Conservancy Press, Zhengzhou, 2015)

24. Yellow River Water Conservancy Committee. Theory and practice of water and sediment regulation of Yellow River (Yellow River Water Conservancy Press, Zhengzhou, 2013)

25. J.H. Zhang, J.Q. Xia, H.B. Ma, et al., Selection and regulation of deposition patterns of Xiaolangdi Reservoir (Yellow River Water Conservancy Press, Zhengzhou, 2016)

26. S.X. Li, J.H. Zhang, J.Q. Xia, et al., Reservoir density current (Yellow River Water Conservancy Press, Zhengzhou, 2013)

27. T. Wang, S.K. Chen, H.B. Ma, et al., J. Sediment Res. 5, 60-66 (2011)

28. T. Wang, X.P. Li, Z.H. Ren, Experimental Research on Water and Sediment Regulation of Middle and Small-scale Floods in 2016 [R]. Zhengzhou: Yellow River Institute of Hydraulic Research. Huang Ke Ji zX-2017-07, 2016:17-27.

29. S.X. Li. J.H. Zhang, S.K. Chen, et al., J. Hydraul. Eng. 5, 567-572 (2006).

30. J.Z. Li. Engineering planning (China Water Power Press, Beijing 2006) 